\title{
Paediatric Recurrent Ear, Nose and Throat Infections and Complications: Can We Do More?
}

\author{
Ricardo Marengo · José A. Ortega Martell · Susanna Esposito
}

Received: November 12, 2019 / Published online: April 24, 2020

(C) The Author(s) 2020

\section{ABSTRACT}

Recurrent respiratory tract infections (rRTIs), of which there are three main groups-otitis media, tonsillitis and sinusopathies-are very common in paediatric populations and are associated with significant morbidity and mortality due to complications. These infections substantially reduce quality of life for paediatric patients and their families and are a significant personal, medical and economic burden on the

Enhanced digital features To view enhanced digital features for this article go to https://doi.org/10.6084/ m9.figshare.11671980.

R. Marengo

ENT and Audiology Department of CEMIC Surgery

Department, University Institute CEMIC,

Valdenegro, Buenos Aires, Argentina

J. A. Ortega Martell ( $₫)$

Department of Immunology, Universidad

Autónoma del Estado de Hidalgo, Pachuca, Hidalgo, Mexico

e-mail: drortegamartell@prodigy.net.mx

J. A. Ortega Martell

Consejo Nacional de Inmunología Clínica y Alergia,

Mexico city, Mexico

J. A. Ortega Martell

Organización Mundial de Alergia (World Allergy

Organization), Milwaukee, WI, USA patients, the patients' families and the healthcare system. Most rRTIs are of viral origin; however, indiscriminate use of antibiotics in their treatment has led to development of bacterial resistance. Effective management of rRTIs to reduce the burden of disease and to avoid overuse of antibiotics has become a great therapeutic challenge. New strategies for the management of paediatric rRTIs include focus on prevention using non-specific immunomodulators to boost the body's natural defences against infection and to downregulate

\section{J. A. Ortega Martell}

Colegio Mexicano de Inmunología Clínica y Alergia

(CMICA), Mexico city, Mexico

\section{J. A. Ortega Martell}

Colegio Mexicano de Pediatras Especialistas en

Inmunología Clínica y Alergia (COMPEDIA),

Colonia Nápoles, Mexico

\section{S. Esposito}

Pietro Barilla Children's Hospital, Milan, Italy

S. Esposito

Department of Medicine and Surgery, University of Parma, Parma, Italy

\section{S. Esposito}

World Association for Infectious Diseases and Immunological Disorders (WAidid), Milan, Italy 
infection- and allergen-induced airway inflammation. The oral immunomodulator, OM-85, a bacterial lysate, acts on the innate and adaptive branches of the immune system, conferring protection against viral and bacterial infections, and controls inflammation, thereby reducing tissue damage. OM-85 has demonstrated good tolerability and clinical efficacy in reducing the number and duration of RTIs in children with recurrent airway infections. It has also been reported to reduce the use of concomitant medications, including antibiotics, time to cure and school absenteeism. OM-85 is efficacious and well tolerated when administered concomitantly with inactivated influenza vaccine (IIV) and has been shown to reduce wheezing attacks induced by RTI in young children. Clinical results show that the greater the risk of rRTIs, the greater the benefit with OM-85. OM85 may be considered a promising tool to add to the limited armamentarium of the ear, nose and throat (ENT) physician dealing with rRTIs and their complications, such as recurrent wheeze and asthma inception.

Keywords: Immunomodulator;

Nasopharyngitis; OM-85; Otitis media; Paediatric; Recurrent ENT infections; Rhinosinusitis; rRTIs; Tonsillitis; URTIs

\section{Key Summary Points}

Recurrent respiratory tract infections (rRTIs) are common in young children and present a significant burden on the patients' families and the healthcare system overall. As a result, effective management of rRTIs becomes a great therapeutic challenge.

Immunological immaturity and environmental factors put children at increased risk of recurrent respiratory tract infections. rRTIs early in life, and episodes of viral-induced wheezing in particular, are a significant risk factor for asthma confirmed diagnosis in later life.
The use of non-specific

immunomodulation (i.e. OM-85) to boost

the body's natural defenses against infection and to downregulate in parallel airway inflammation and airway hyperreactivity offers a strategy in the management of rRTIs in children, including those with atopy.

The efficacy and safety of oral bacterial lysates OM-85 have been assessed in a number of clinical studies in children and may be considered an effective preventative strategy to protect children against recurrent ENT infections and improve the disease burden for the patients and their families.

\section{INTRODUCTION TO RECURRENT RESPIRATORY TRACT INFECTIONS}

Respiratory tract infections (RTIs) of the upper airways (URTIs) comprise approximately $90 \%$ of all infections and include rhinitis (nasopharyngitis and rhinosinusitis), tonsillitis (amygdalitis), laryngitis and otitis media [1-3]. These infections are very common in paediatric populations and are associated with significant morbidity and mortality, particularly in developing countries, and are often recurrent (rRTIs). The practical definition of rRTI is at least three episodes of a specific ear, nose and throat (ENT) infection per year, but there is no overall consensus about the threshold of recurrence in all ENT infections. Acute otitis media (AOM) is defined as recurrent when there are three or more episodes in 6 months or four or more episodes in a year [4]. In the case of chronic rhinosinusitis (CRS) with acute exacerbations, recurrence is defined as five or more episodes in a year. The definition of recurrent tonsillitis is five episodes per year in the first year of recurrence or three or more episodes per year for 3 consecutive years [5]. rRTIs are very common in otherwise healthy children. In developed countries, up to $25 \%$ of children aged under 1 year and around $18 \%$ aged $1-4$ years experience rRTIs [6]. Typical incidence rates are 3-8 
URTIs per year, with around $10-15 \%$ of children experiencing at least 12 infections each year [7]. At least $80 \%$ of RTIs are caused by viruses [6], with the most common including human rhinovirus (HRV), respiratory syncytial virus (RSV), adenoviruses and influenza virus $[6,8]$. When these infections are recurrent (rRTIs), they reduce the quality of life for both children and their families. A study by Jiang et al. in 420 children aged $2-4$ years (220 children with rRTIs, 200 healthy children) showed that children with rRTIs had statistically significantly $(p<0.05)$ lower physical $(84.2 \pm 12.2$ vs. $92.6 \pm 6.8)$, emotional $\quad(63.4 \pm 13.7 \quad$ vs. $88.7 \pm 13.3)$, social $(79.7 \pm 13.0$ vs. $95.4 \pm 8.0)$ and school functioning $(67.9 \pm 7.1$ vs. $89.5 \pm 8.0$ ) than healthy children according to the Generic Core Scales score; see Fig. 1 [9].

Furthermore, rRTIs have a socioeconomic impact (frequent medical visits [10], healthcare costs [6], transport costs, work/school absenteeism [11]) and are associated with deteriorated lung function and decreased quality of life of the children and their families [9, 12]; see Fig. 2.

Another important consideration in paediatric patients with rRTIs is antibiotic collateral damage (development of bacterial resistance and microbiota disruption) as a result of indiscriminate use of antibiotics [13]. Effective management of rRTIs to reduce the burden of disease and to avoid overuse of antibiotics for these infections of mostly viral origin has become a great therapeutic challenge.
This article summarises the latest research into paediatric rRTIs presented at the satellite symposium "Pediatric recurrent ENT infections: can we do more?" at the First World Congress of Pediatric ENT ("In the 21st century"), held in Buenos Aires, Argentina, on 8 April 2019.

\section{METHODS}

This article is based on previously conducted studies and does not contain any studies with human participants or animals performed by any of the authors. No systematic research of the literature was performed but a review of recent literature was conducted and the most relevant articles were retained.

\section{Protection of an Immunomodulator Against Airways Infections in Paediatrics}

\section{The Rationale for Using OM-85 Centres on the Gut-Lung Immune Axis}

The human immune system comprises a myriad of cells and molecules that need to work in coordination like a finely tuned orchestra, communicating with each other and the rest of the body cells, with multiple cytokines acting as molecular messengers. The human immune system can be subdivided into three branches: constitutive, innate and adaptive immunity. Innate immunity can be broadly viewed as a rapid (minutes to hours), non-specific response

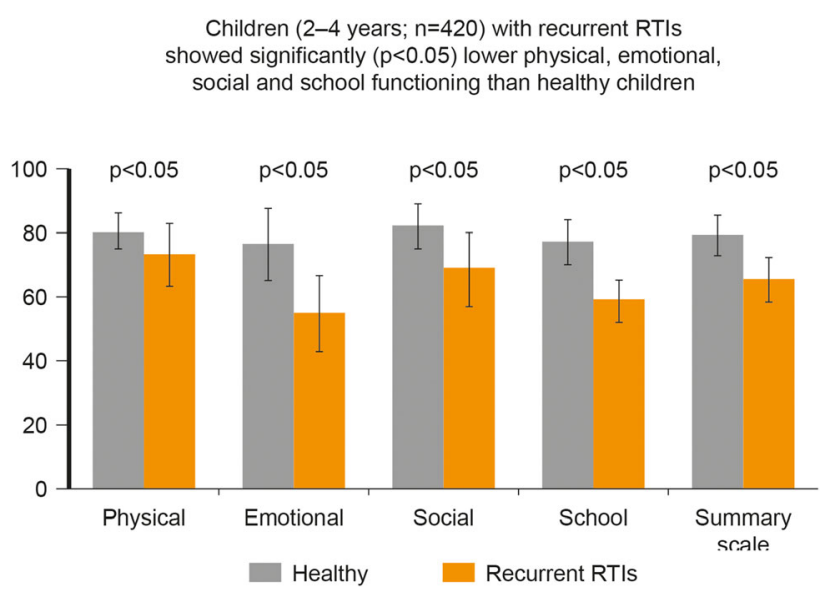

Fig. 1 Impact of rRTIs on quality of life. Reference: Jiang et al. [9] 


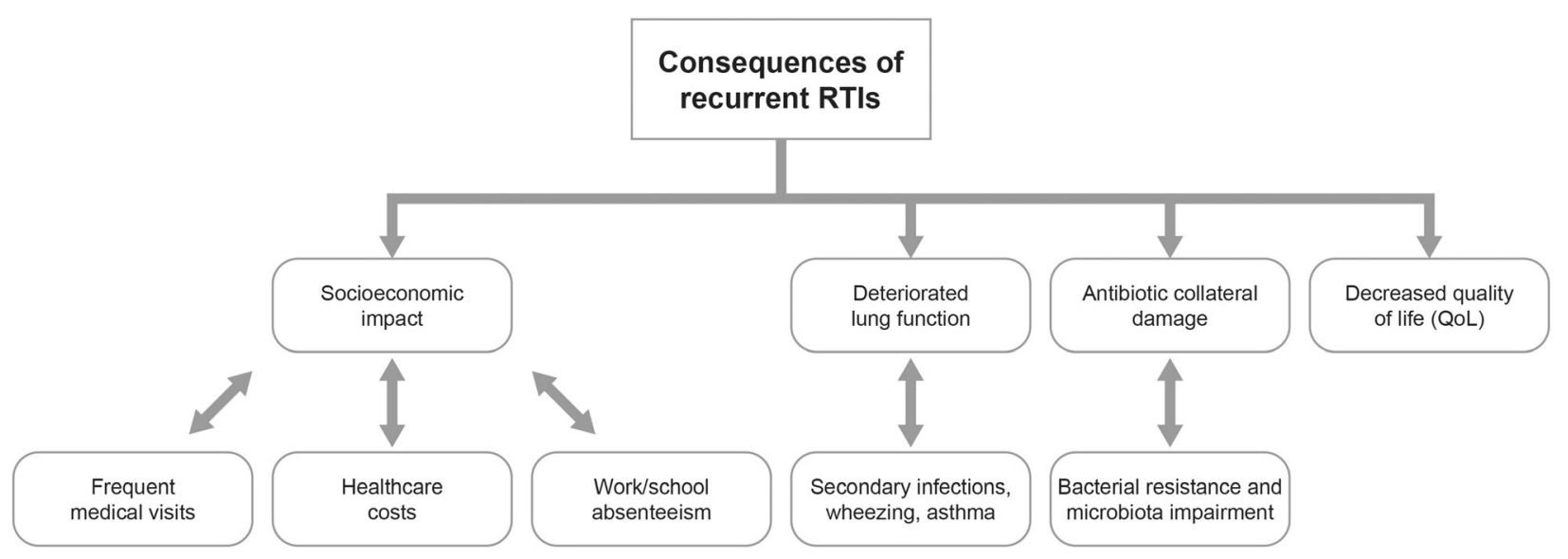

Fig. 2 Burden of disease: consequences of recurrent RTIs. Reference: Adapted from Schaad et al. [4]

to a wide variety of stimuli, with no memory function. In contrast, adaptive immunity provides a slower (days to weeks), specific and adjustable response to antigens that leaves a memory, which enables the immune system (Tand B-lymphocytes) to respond faster and stronger the next time it is challenged by the same antigen.

OM-85 is an oral immunomodulator the active principle of which is an extract of 21 bacterial strains of 5 different pathogenic respiratory bacterial genera (8 species and subspecies). Non-specific immunomodulation with bacterial lysate OM-85 to boost the body's natural defences against infection and to downregulate infection- and allergen-induced airway inflammation and airways hyper-reactivity provides a strategy for the management of rRTIs in at-risk (including atopic) children [14-16].

The mechanistic rationale for using OM-85 to prevent respiratory conditions centres on the gut-lung immune axis. When administered orally, OM-85 diffuses through the intestinal mucosa into Peyer's patches [organised lymphoid follicles of the gut-associated lymphoid tissue (GALT)], selectively activates dendritic cells (DCs) and shapes the innate and adaptive immune response. The active components of OM-85 are sampled by antigen-presenting cells (APCs; e.g. DCs) resident in the Peyer's patches, and this leads to maturation of DCs (together with B-cells) into an APC phenotype (upregulation of CD40, CD80 surface expression) [18].
The subsequent DC-initiated immune cascade involves homing of cells from both innate and adaptive branches of the immune system to the mucosal-associated lymphoid tissue (common examples are oral, airway, and intestinal mucosa [17]) and subsequent polyclonal antibody production [18-22].

OM-85 affects innate immunity through increasing antimicrobial peptides (AMPs); increasing neutrophil recruitment through chemokines (CCL2, CCL3, CXCL1, CXCL5, CXCL6, CXCL8); increasing natural killer cells (cytotoxic lymphocytes), monocytes and neutrophils, which are important for phagocytosis; and increasing antiviral cytokine release (e.g. interferon [IFN]- $\alpha$ and IFN- $\beta$ ) [18-20].

In adaptive immunity, OM-85 increases the interaction between T-cells and dendritic cells and increases cytotoxic T-cells and production of IFN- $\gamma$. It also affects how B-cells produce antibodies, particularly immunoglobulins (Igs) IgG and IgA (IgA is the best antibody defence in salivary and airway secretions), and increases B cell maturation [18].

\section{OM-85 Confers Protection Against Viral and Bacterial Infections}

The studies in this section were performed in vitro and in vivo; therefore, $n$ represents the number of repeated experiments.

OM-85 acts on the innate and adaptive immune branches, conferring protection against viral and bacterial infections. OM-85 


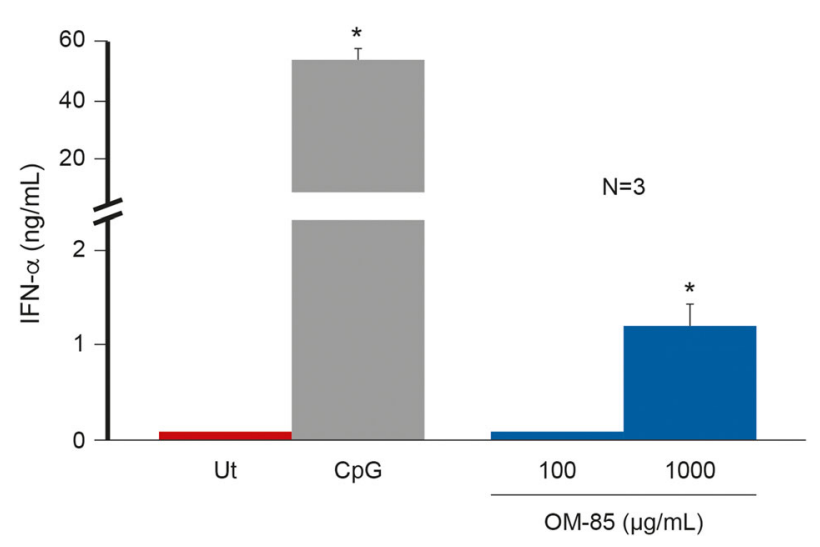

Fig. 3 Effect of OM-85 on IFN- $\alpha$ production. ${ }^{*} p<0.05$. $C P G 5^{\prime}$-C-phosphate-G-3, Ut untreated. Reference: Parola et al. [19]

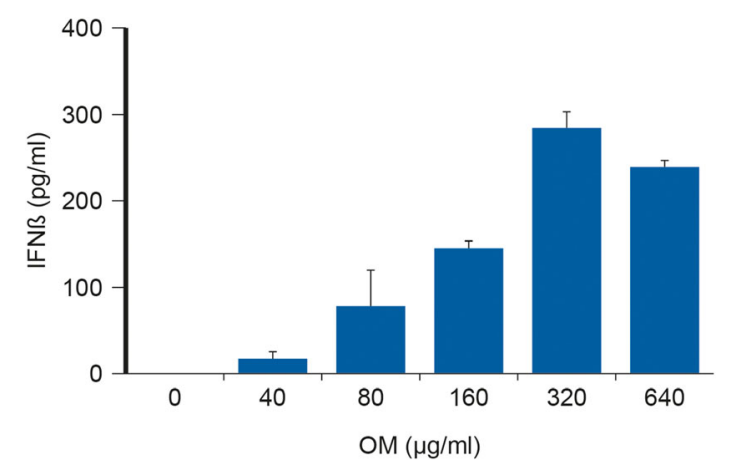

Fig. 4 Effect of OM-85 on IFN- $\beta$ production at different concentrations ( 40 to $640 \mathrm{mcg} / \mathrm{ml}$ ). The increase on IFNbeta production correspond with the increasing concentration of OM-85. ${ }^{* * *} p \leq 0.001$. These in vitro results represent mean $\pm \mathrm{SD}(n=3$ technical replicates $)$. Reference: Parola et al. [19]

increases the expression of key antiviral cytokines (IFN- $\alpha$, IFN- $\beta$ and IFN- $\gamma$ ), thereby helping to create a basal antiviral state; see Figs. 3, 4 [20-22].

Further mechanisms by which OM-85 increases the antiviral immune response include: increasing human $\beta$-defensins (antimicrobial defence and cell surface receptors), increasing peripheral blood mononuclear cells (PBMC) and release of non-specific polyclonal antiviral response (RSV and influenza virus antibodies) in the serum and airways. OM-85 has been shown to lower viral load in lung tissue on day-5 post-influenza infection compared with an untreated control group, providing rapid control of infection in a well-defined in vivo infection model [23] (see Fig. 5) and to reduce HRV infection in control and asthma human bronchial epithelial cells.

OM-85 also demonstrates an antibacterial effect (e.g. against Streptococcus pneumoniae or Klebsiella pneumoniae) and protects against secondary bacterial infections [23]; see Fig. 6. OM85 increases the antibacterial immune response by releasing non-specific polyclonal IgA and IgG in serum and airways and improving disease scores in animal models following influenza challenge [21, 23].

\section{Downregulation in Chronic Inflammatory State}

Effective treatments for rRTIs need to provide an antiviral state and have an antibacterial effect so the patient avoids common viral respiratory infection and secondary bacterial respiratory complications to reduce the overall associated morbidity. The treatment should control inflammation to reduce tissue damage, thus providing a two-stage process of combat and control.

As well as creating a pre-alert condition in the infection state, OM-85 has been shown to downregulate (dampen) the immune system to help in airway chronic inflammatory states, such as CRS [24, 25], by decreasing the levels of proinflammatory cytokines (e.g. interleukin [IL]-1 $\beta$ ) in a dose-dependent manner [24], dampening recruitment of inflammatory cells 


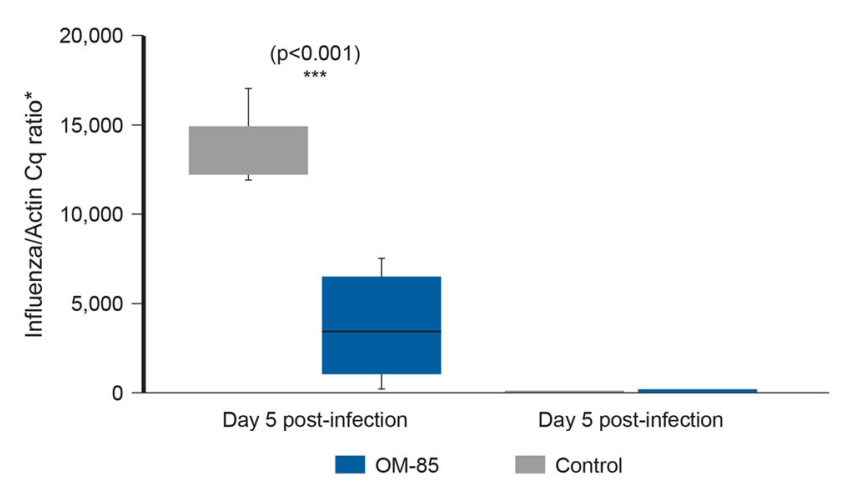

Fig. 5 Effect of OM-85 against viral infections in an in vivo infection model. The viral load in lung tissue was determined on day 5 and 10 post-influenza virus infection. Data are representative of 2-5 experiments with
5-10 mice per time point. Error bars represent minimum to maximum value range. Statistical analysis was performed by Student's $t$ test. ${ }^{* * *} p<0.001$. Reference: Pasquali et al. [21]

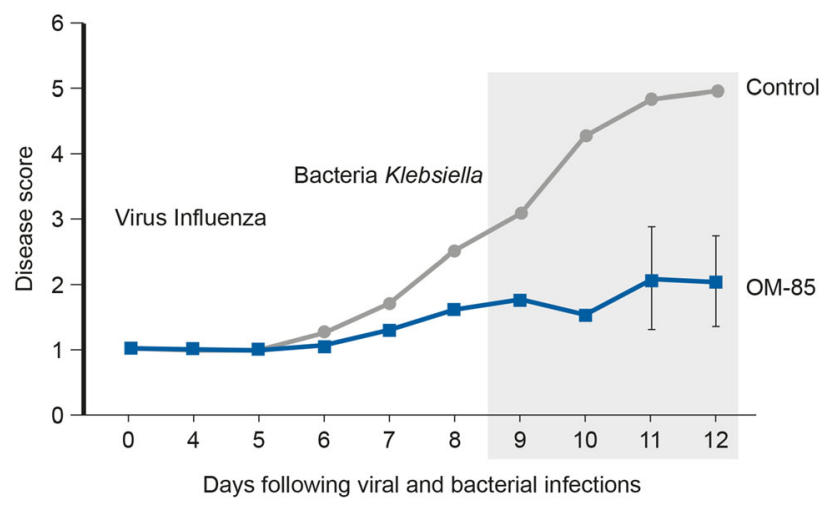

Fig. 6 Effect of OM-85 against secondary bacterial infections in an in vivo infection model. Disease score of control or OM-85-treated mice following influenza infection and Klebsiella pneumoniae. Data are representative of

[22] and increasing the levels of anti-inflammatory cytokines (e.g. IL-10) [20], thus reducing tissue damage. Other effects of OM-85 include increasing tolerogenic dendritic cells $\left(\mathrm{CD} 103^{+}\right)$, activation of T-cells or conversion to Treg cells, and decreasing type 2 DCs (ICOS-ligand) $[18,21]$.

OM-85 may also aid maturation of the immune system in children by correcting Th1/ Th2 imbalance through increasing Th1 cytokines (IFN- $\gamma$ ), increasing Treg cytokines (IL-10) and decreasing Th2 cytokines (IL-4, IL-5, IL-13) $[18,24]$. The correction of this Th2-oriented imbalance and other anti-inflammatory activity three experiments with 5-10 mice per time point. Error bars represent SD. Reference: Pasquali et al. [21]. https:// creativecommons.org/licenses/by-nc/4.0/

(such as decreasing inflammatory cell infiltration and decreasing eosinophils, neutrophils, macrophages and T- and B-cells [18]) may help reduce atopic responses related to wheezing and asthma. For example, increased levels of IL-10 are associated with decreased airway inflammation, subsequent tissue remodelling and decreased hypersecretion. These effects combined with the reduced risk of rRTIs, which predispose to asthma and cause exacerbations, form the mechanistic framework for a reduced risk of these conditions $[16,18]$. 


\section{NEW INSIGHTS IN PREVENTING RECURRENT EAR, NOSE AND THROAT INFECTIONS IN CHILDREN}

\section{Unmet Need in Management of Recurrent Respiratory Tract Infections in Children}

The current standard of care in management of rRTIs in children is primarily focused on RTIs treatment including symptom relief $[26,27]$. Ideally, the focus should be on prevention rather than treatment of RTIs and this should be based on a clear understanding of risk factors. There is an important unmet need for prompt and correct diagnosis as well as an intelligent use of multiple and complementary preventative approaches to ultimately reduce the overall burden of disease and the overuse and abuse of antibiotics for these mostly viral RTIs [4].

\section{Need for Preventative Strategies}

General prophylaxis and other common preventative measures are put in place to reduce the infection rate in this fragile paediatric population. Prevention of recurrences through elimination of environmental risk factors and patient-tailored behavioural modification are strongly recommended $[6,28]$. Unfortunately, these measures are only partially effective; behavioural interventions (e.g. avoidance of risk factors) are not always applicable or followed. Alternative strategies include targeted medical interventions (such as systematic use of available paediatric vaccination programmes, when indicated or recommended) and surgical interventions (e.g. adenotonsillectomy in cases of recurrent pharyngo-tonsillitis), although the indications are limited and potential risks associated with surgical procedures are an important consideration [5].

\section{Efficacy of OM-85 in Children}

Boosting immune system activity with nonspecific immunomodulation in paediatric patients has been suggested as a potential complementary strategy to improve protection of children against rRTIs [18]. Oral bacterial lysate OM-85 has demonstrated clinical efficacy in reducing RTIs in at-risk children with rRTIs [29-32]. The dose regimen for OM-85 used in 6or 12-month studies in prevention of RTIs is 10 days/month for 3 months.

In children affected by rRTIs in a randomised, double-blind, placebo-controlled study, the proportion of RTI-free patients (the primary endpoint) was significantly higher in the OM-85-treated group compared with the placebo group (see Fig. 7) with total infections $35 \%$ lower with OM-85 versus placebo in all children in the study and $28 \%$ lower in children aged $<6$ years [33].

OM-85 has also been associated with fewer RTIs than placebo (143 versus 299; difference of $52 \%$; $p<0.001$; primary endpoint) at 6 months in a randomised, double-blind, placebo-controlled study in girls aged $6-13$ years [35]. OM85 also reduced the number of infections in each patient from 494 to 143 , with placebo reducing the number of infections from 509 to $299(p<0.001$, for both comparisons) [35]. There were fewer infections at each month with OM-85 compared with placebo $(p<0.05$, at months 2, 3, 4 and 6) [35].

OM-85 has also been reported to reduce antibiotic use, time to cure and school absenteeism; see Fig. 8 [35].

OM-85 was also shown to significantly reduce the number and duration of acute RTIs (primary endpoint), with 131 acute RTIs in paediatric patients who received OM-85 compared with 224 in those who received placebo in a randomised, double-blind, placebo-controlled study [36]. The mean RTIs per patient were significantly lower with OM-85 at 12 months: 5.0 vs. 8.0 ( $p<0.001), 38 \%$ lower (all patients) and 4.9 vs. $8.3(p<0.01), 41 \%$ lower (patients aged under 6 years). The total duration of acute RTIs was significantly lower with OM-85 compared with placebo: median 30.5 vs. 55.0 days $(p<0.001), 45 \%$ lower (all patients). A higher proportion of children (57\% higher; $p<0.001)$ without recurrences (fewer than six acute RTIs) and less antibiotic consumption (44\% less; $p<0.001)$ was also reported with OM-85 [36]. 


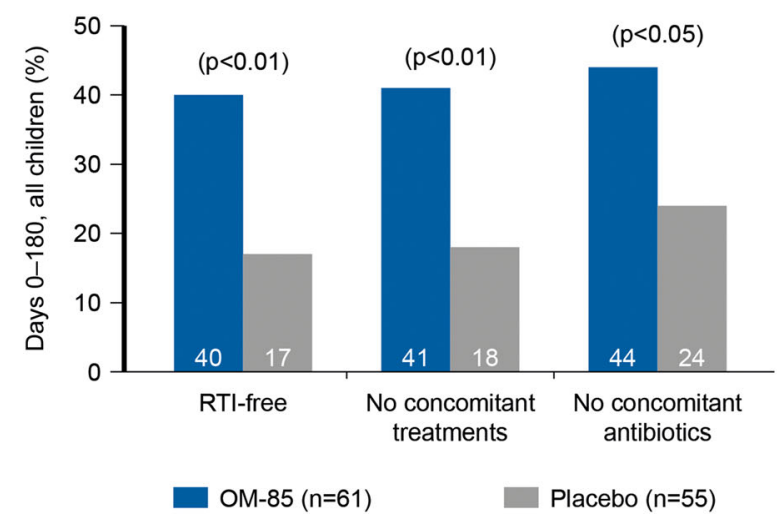

Fig. 7 Effect of OM-85 on incidence of RTI and use of concomitant treatments in paediatric patients with rRTIs. Reproduced with permission from Paupe Jean. Immunotherapy with an Oral Bacterial Extract (OM-85

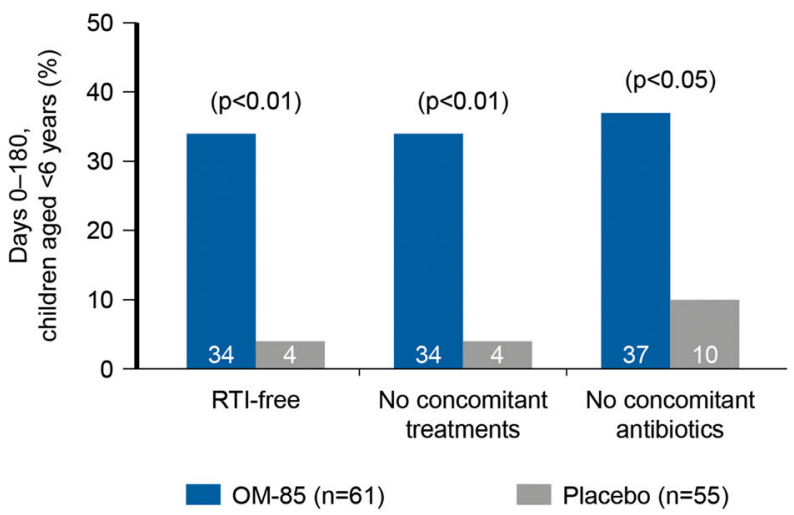

BV) for Upper Respiratory Infections. Respiration. 1991; 58:150-154. Copyright(C) 1991 Karger Publishers, Basel, Switzerland
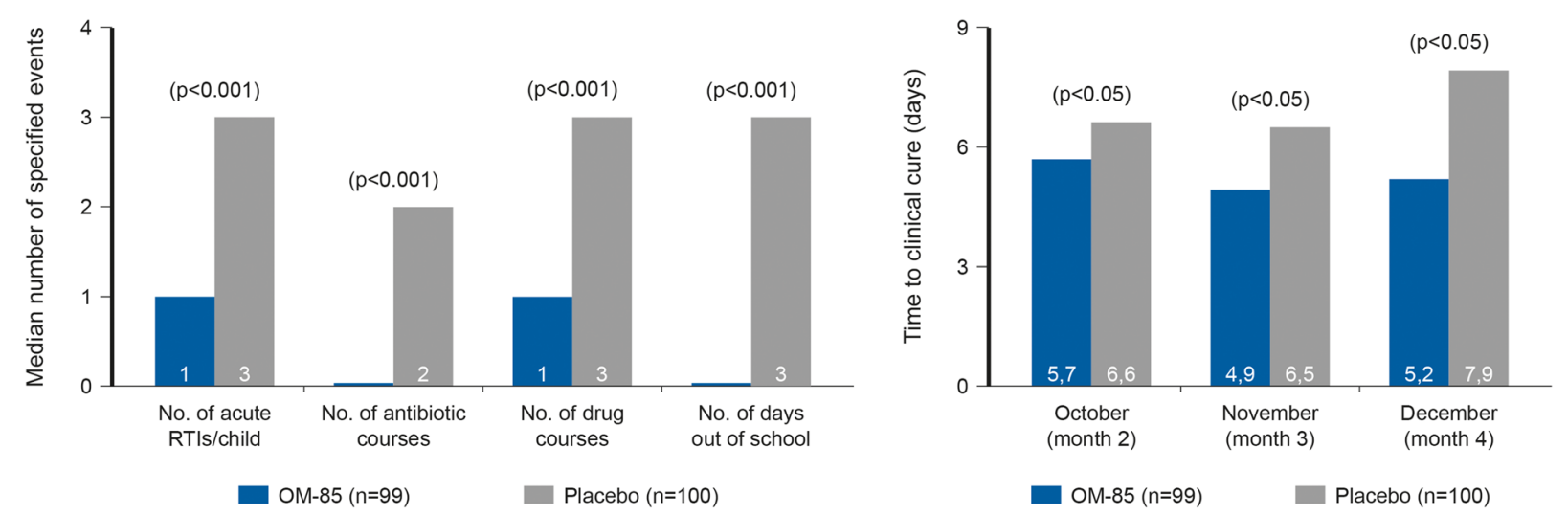

Fig. 8 Effect of OM-85 on antibiotic use, time to cure and school absenteeism in paediatric patients with rRTIs. Number of patients: OM-85 group, $n=99$; placebo group, $n=100$. Study duration was 6 months. Reprinted from Clinical Therapeutics, 22, Jaime V. Jara-Pérez,

Almost a decade ago, Prof. Schaad and the Cochrane collaboration published two independent meta-analyses of several paediatric randomised controlled trials where children treated with OM-85 had significantly and consistently fewer cases of rRTIs [33, 34]. In the meta-analysis published by Schaad, a 35.5\% decrease in the overall mean number of paediatric acute RTIs was demonstrated with OM-85 compared with placebo [37]. This was confirmed in 2012 in an updated meta-analysis in

Arturo Berber, Primary prevention of acute respiratory tract infections in children using a bacterial immunostimulant: A double-masked, placebo-controlled clinical trial, 748-759, Copyright (2000) with permission from Elservier

852 children performed by the Cochrane collaboration group in which the immunomodulator OM-85 was shown to significantly reduce total acute RTIs by $35.9 \%$ [38].

\section{Concomitant Use of OM-85 and Inactivated Influenza Vaccine}

The concomitant use of OM-85 $3.5 \mathrm{mg} /$ day and inactivated influenza vaccine (IIV) showed superior clinical benefit compared with IIV only 
in a prospective, randomised, single-blind study in 68 children aged 36-59 months with rRTIs and at least one previous IIV [35]. OM-85 was administered once daily for 10 days during 3 months, and IIV was administered 15 days after the end of the first 10-day course. There was a greater reduction in lower RTIs (67\%; $p<0.05$ ), URTIs (35\%; $p<0.05$ ), antibiotic use (72\%; $p<0.05)$ and days absent from school (52\%; $p<0.05)$ in the OM-85 plus IIV group compared with the IIV only group. These results show OM-85 may be administered with IIV to reduce rRTIs in this at-risk paediatric population and does not affect humoral immunity to the vaccine. Follow-up for 14 days after vaccination with IIV given with or without OM-85 showed that OM-85 administered with IIV was well tolerated in the short term [39]. Local and systemic adverse events were similar in both groups, and no serious adverse events were reported.

\section{OM-85 and Virus-Induced Wheezing}

Children with rRTIs often suffer from recurrent wheezing, which is a significant risk factor for the development of asthma in later life. In a doubleblind, randomised, placebo-controlled, parallelgroup study in 75 children aged 1-6 years with recurrent nasopharyngitis and virus-induced wheezing, OM-85 $3.5 \mathrm{mg} /$ day prophylaxis (OM85 given 10 days at the start of three consecutive months) led to significantly fewer episodes of nasopharyngitis $(37.5 \% ; p<0.001)$ and significantly decreased the occurrence of wheezing attacks (37.9\%; $p<0.001$; primary endpoint) and the duration $(p<0.001$ for both cumulative attack duration and duration of a single attack) compared with placebo; see Fig. 9 [36].

The impact of treatment observed after 3 months was maintained for 12 months after the prescription, which shows OM-85 prevents wheezing attacks in young children [40]. Reduction in recurrent nasopharyngitis was also first observed at 3 months and persisted until month 12 .

A recent meta-analysis by Yin confirmed the clinical benefit of OM-85 compared with no treatment in 4851 patients from several studies in terms of decreasing the duration of wheezing [37].

Recent data from a retrospective, observational study in 400 children aged 3-6 years (200 received OM-85, 200 no treatment) with rRTIs indicate that OM-85 administered before the winter season can effectively and safely reduce the risk of new episodes of RTI (reduction of wheezing episodes and antibiotic courses) in this paediatric population and that a second course of OM-85 administration the following year can be useful to maintain protection, particularly when the diagnosis of rRTIs is made in younger children whose immune systems still require considerable time to mature [38]. OM-85 was well tolerated in this study: no severe adverse events were reported during either OM-85 course. The results also confirmed the safety profile of OM-85 when administered with IIV $(n=49)$.

A randomised, double-blind, placebo-controlled study to assess the efficacy and safety of OM-85 in 288 children aged 1-6 years with rRTIs showed that OM-85 can significantly reduce the risk of new infections (primary endpoint) in these patients, thus limiting the total burden of these diseases regardless of atopy/allergy history [39]. During the study period, at least one new episode of RTI was diagnosed in over $65 \%$ of children who were given placebo and in only about one-third of those who received OM-85; efficacy was greater in children at high risk of RTIs (50\% of placebo patients had at least three episodes of nasopharyngitis/pharyngitis compared with $21 \%$ of OM-85 patients), and recurrences in treated children were reduced by about $50 \%$ compared with untreated patients.

\section{LESSONS LEARNT FROM CLINICAL PRACTICE IN RECURRENT ACUTE OTITIS MEDIA, CHRONIC RHINOSINUSITIS AND ACUTE TONSILLITIS}

\section{Challenges of Paediatric Recurrent ENT Infections in Clinical Practice}

Approximately 90\% of children will experience AOM before they reach school age, with half of 


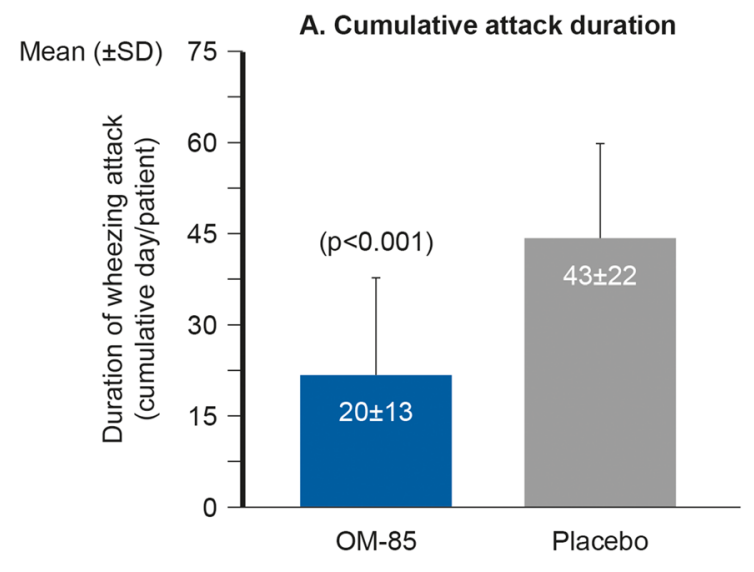

Fig. 9 Effect of OM-85 on cumulative wheezing attack duration and duration of a single wheezing attack in paediatric patients with rRTIs. Reprinted from Journal of Allergy and Clinical Immunology, 126, Cem Hasan Razi, Koray Harmanc1, Ayhan Abacı, Osman Özdemir, Şamil

them already presenting with infection during the first year of life [40]. Up to 5\% of children with URTIs progress to acute rhinosinusitis, and some may develop chronic respiratory illness, such as CRS [41]. Tonsillitis is also very common during childhood and recurrence may be an indication for tonsillectomy (amygdalectomy), although the risks associated with the procedure should be considered.

\section{Recurrent Acute Otitis Media}

To treat paediatric patients with recurrent acute otitis media (rAOM) effectively, ENT physicians need to consider the patients as a whole, including medical, social and environmental factors. Factors and habits that influence the incidence of $\mathrm{rAOM}$ include oral ventilation, breastfeeding, pacifier and milk bottle use, passive smoking, attendance at childcare institutions, chlorine and algaecides, socioeconomic factors and crammed households. Pharmacological treatments comprise a diverse set of tools, including hypertonic saline solutions, topical corticoids, antihistamines, non-specific immunomodulation, specific influenza vaccine (in children aged at least 6 months), pneumococcal vaccine, specific immunoallergic agents, gastroesophageal reflux medicine and

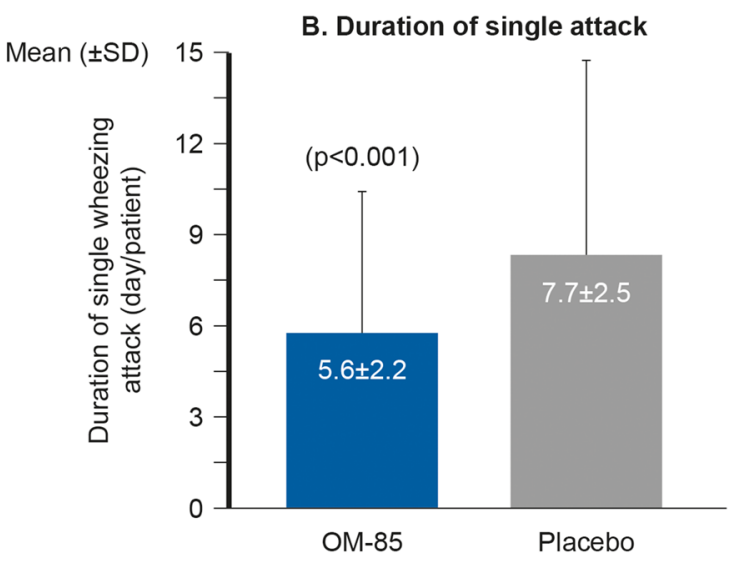

Hizlı, Rahime Renda, Fersin Keskin, The immunostimulant OM-85 BV prevents wheezing attacks in preschool children, 763-769, Copyright (2010), with permission from Elservier

prophylactic antibiotics (in special cases of immune deficiency). Other associated treatments for this condition include phoniatric treatment for ventilation, swallowing and Eustachian tube function, and orthopaedic or orthodontic treatment.

The increasing scientific evidence for immunomodulation in prevention of rAOM has led to a shift in focus from treatment to prevention in the management of paediatric patients with rAOM. The efficacy and safety of the bacterial lysate OM-85 have been assessed in several paediatric clinical studies.

Although only a small number of cases of otitis media were reported in the children in two randomised, placebo-controlled studies (8/ 99 and 25/100 [35] and 3/26 and 14/28 [36] in the OM-85 and placebo groups, respectively), substantially fewer $(68-75 \%$; $p<0.001)$ AOM episodes were observed in the patients treated with OM-85 compared with those who received placebo $[35,36]$.

A retrospective intervention study in 100 children aged $<13$ years with confirmed $\mathrm{rAOM}$ showed that OM-85 (3.5 mg/day) was associated with no co-morbidities in $81 \%$ of patients (the remaining $19 \%$ had craniofacial co-morbidities or laboratory-confirmed immunoallergic pathologies) and 94\% (76 of 81) of patients 
adhered to treatment (administration on an empty stomach was required and there was greater adherence when administration was straight after school rather than first thing in the morning) [42]. A total of 61 of $76(80 \%)$ treatment-compliant paediatric patients did not present with $\mathrm{rAOM}$ after OM-85 treatment (comparing the number of AOM episodes per year before treatment with that after treatment in each patient). Furthermore, $49 \%$ of paediatric patients did not require antibiotic therapy during the first 6 months, and 34\% did not require antibiotic therapy in the first 12 months. The study results showed that atopic patients, who are more susceptible to complications such as rAOM, may benefit further from immunomodulatory intervention and confirm the further clinical benefit of such intervention in these atrisk children.

\section{Chronic Rhinosinusitis}

Immunomodulation with OM-85 has also demonstrated benefits in paediatric patients with CRS. In a randomised, double-blind, placebo-controlled trial including 55 children aged 4-12 years with acute exacerbation of CRS, OM85 significantly decreased the number (by 65\%) and the total duration (by 64\%) of acute episodes after 6 months compared with placebo [27]. Historically, symptom improvement due to prevention of acute episodes is usually seen by the 2nd month of treatment; here, symptoms such as cough, nasal discharge and nasal congestion improved after 15 days of treatment, with the improvement lasting and increasing into the 5th month after the start of treatment and at the end of the study (6th month) for cough and nasal congestion symptoms; see Fig. 10.

Significantly fewer $(65 \% ; p<0.05)$ and shorter $(73 \% ; p<0.01)$ episodes of CRS were also noted. In addition, serum IgA levels were significantly greater with OM-85 than placebo at month $5(p<0.01)$ and $6(p<0.05)$.

\section{Subacute Rhinosinusitis}

Another randomised, double-blind, placebocontrolled study in 56 children aged 18 months to 9 years with subacute rhinosinusitis demonstrated a more rapid time to improvement/cure and more pronounced clinical improvement (primary endpoint) with OM-85 $(3.5 \mathrm{mg} /$ day for the first 10 days of 3 consecutive months) compared with placebo (time to improvement/cure $44 \%$ vs. $24 \% ; p<0.05$ ) and consequently reduced total days of illness [43].

As well as reducing the number of exacerbations, OM-85 significantly reduced the use of concomitant medications (21\%; $p<0.05)$, including antibiotics (29\%; $p<0.05)$, compared with placebo. Duration of concomitant treatment was also reduced with OM-85 compared with placebo $(24 \% ; p<0.05)$.

The difference in clinical score between the OM-85 and placebo groups was seen from day 1 . The most pronounced effect on clinical score was observed since day 15 and was maintained at a statistically significant level over time.

\section{Acute Tonsillitis}

In a retrospective, observational study in 177 children aged 1-15 years with recurrent acute tonsillitis, OM-85 $(3.5 \mathrm{mg} /$ day for the first 10 days of 3 consecutive months) decreased the frequency of episodes of acute tonsillitis in the short term and reduced recurrent tonsillitis over the long term [44]. After a median of 9 months' follow-up, no tonsillectomy was required in $>$ $50 \%$ of treated patients [47]. A total of $75.6 \%$ patients treated and followed up responded to treatment [total response (no new episodes or recurrent tonsillitis), 51.2\%; partial response (reduced re-exacerbation), 24.4\%] indicating a significant reduction in recurrent tonsillitis.

\section{Safety and Tolerability of OM-85}

OM-85 has shown a good safety profile that has remained unchanged in nature and frequency for over 30 years of clinical paediatric use, so is supported by long established use and robust medical evidence. Adverse events observed during OM-85 treatment are mainly non-serious and transitory, with known and manageable risks [37, 38]; the most common are headache, gastrointestinal symptoms and skin rashes.

In a prospective study of children with IgA deficiency and febrile attacks, treatment with 

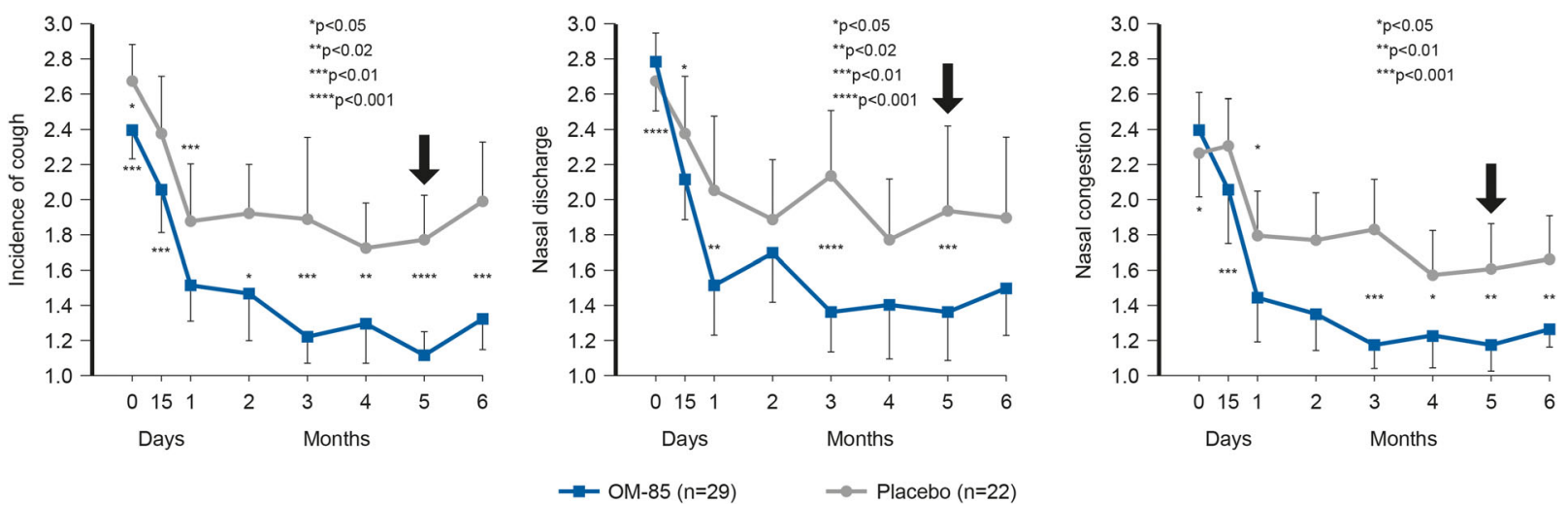

Fig. 10 Effect of OM-85 on symptoms of chronic rhinosinusitis in paediatric patients. Presence by a 3-point rating scale: $1=$ none; 2 = moderately frequent (weekly); $3=$ frequent (daily). Assessment by a 4-point rating scale: $0=$ none; 1 = clear; $2=$ mucoid; $3=$ purulent. Frequency and intensity as: severe congestion: $1=$ none; 2.5 = moderately frequent (weekly); 3 = frequent (daily);

the immunomodulator OM-85 did not affect clinical or laboratory markers for autoimmunity [45], and it was effective and well tolerated in children with mild IgG deficiency [34]. OM-85 was also well tolerated when administered in combination with IIV, did not interfere with antibody titres of vaccine and had no observed effect on humoral immunity [39].

\section{DISCUSSION AND CONCLUSIONS}

Some benign RTIs are expected during immune system development; however, immunological immaturity and environmental factors, including frequent social contact, exposure to pollution and lack of breastfeeding, are associated with increased risk of rRTIs in children. These recurrent infections early in life, particularly episodes of viral-induced wheezing, are a significant risk factor for the development of asthma in later life.

The oral immunomodulator, OM-85, acts on the innate and adaptive branches of the immune system, reducing the risk for against viral and bacterial infections, and controls inflammation, thereby reducing tissue damage. OM-85 has demonstrated good tolerability and mild congestion: $1=$ none; $1.5=$ moderately frequent (weekly); 3 = frequent (daily). Reproduced with permission from Zagar, Sasa and Löfler-Badzek, Dagmar. Broncho-Vaxom ${ }^{\circledR}$ in Children with Rhinosinusitis: A DoubleBlind Clinical Trial. ORL. 1988; 50: 397-404. Copyright $\subseteq 1988$ Karger Publishers, Basel, Switzerland

clinical efficacy in reducing the number and duration of RTIs in children. It has also been reported to reduce the use of concomitant medications, including antibiotics, time to cure and school absenteeism. OM-85 is efficacious and well tolerated when co-administered with IIV and has been shown to reduce infectioninduced wheezing attacks in young children. The downregulating effect of OM-85 may contribute to its good tolerability profile in children with this recurrent condition.

The concomitant use of OM-85 with routine paediatric vaccines, including IIV, is an important consideration. Bearing in mind the mechanism of action of the product and the longterm use in children in clinical practice, and based on pharmacovigilance data, no drug-drug interactions and safety issues with paediatric vaccines have been reported with the use of $\mathrm{OM}-85$. The product is registered in many countries and the label does not report any contraindications on its use with paediatric vaccines.

Meta-analysis of studies with available databases indicate that immunomodulators are not very effective in the prevention of an occasional acute RTI but are in the prevention of recurrent infection [37, 38, 46]. Furthermore, the meta- 
analysis by Schaad showed that the beneficial effect of OM-85 is proportional to the number of RTIs in the previous 12 months and is greater in younger children [37]. Hence, the greater the risk of rRTIs is, the greater the benefit with OM85. Recurrence of RTIs is very common in the first few years of life. OM-85 is associated with extremely relevant benefits in the first 6 years of life [47].

OM-85 provides a pre-alert state, with early protection against viral and bacterial infection, and then regulates inflammation to minimise tissue damage to the patient thereby achieving a balanced immune response.

OM-85 has been recommended as adjunct medication to standard medical treatment in adults with CRS without polyps [48, 49] and was mentioned in a recently published consensus paper as an effective product to prevent RTIs in children [50]. A recent systematic review concluded that OM-85 can be considered a promising and additional tool in the limited armamentarium of the ENT physician dealing with paediatric ENT infections and their complications [51].

A clear understanding of the immune system and the generation of high-quality research data on the use of immunomodulatory strategies are key to progress the treatment of rRTIs in paediatric patients. Recurrence of RTIs is extremely common in the first few years of life and there is considerable clinical evidence to support OM85 as an attractive option for the treatment of paediatric rRTIs, particularly as there are no alternative effective immunomodulators that can be administered in the first years of life.

The evidence of the clinical efficacy and safety of OM-85 in paediatric patients with rRTI (including concomitant use with paediatric vaccines) is sufficiently robust (for example, see [43]) to recommend further studies in children at higher risk who may benefit further from OM-85.

Of course, using modulators is not the only way to reduce unnecessary antibiotic use for rRTIs. The importance of educative antimicrobial stewardship efforts for improving appropriateness of prescriptions cannot be underestimated.

\section{ACKNOWLEDGEMENTS}

Funding. The symposium was organized during the First World Congress of Paediatric ENT on 6-10 April 2019 and was funded by OM Pharma Switzerland, a Vifor Pharma Group company. The Rapid Service Fees were also funded by OM Pharma Switzerland.

Editorial Assistance. Editorial assistance in the preparation of this article was provided by Dr Brigitte Scott of MarYas Editorial Services. Support for this assistance was funded by $\mathrm{OM}$ Pharma Switzerland, a Vifor Pharma Group company.

Authorship. All named authors meet the International Committee of Medical Journal Editors (ICMJE) criteria for authorship for this article, take responsibility for the integrity of the work as a whole, and have given their approval for this version to be published.

Disclosures. Ricardo Marengo declares that he is a speaker contracted by OM Pharma Ltd. José Antonio Ortega Martell declares that he is an active member of the Mexican College of Allergy and Clinical Immunology (CMICA), the National Confederation of Mexican Pediatricians (CONAPEME), the American Academy of Allergy, Asthma and Immunology (AAAAI) and the World Allergy Organization (WAO), COMPEDIA, CONICA and SLAAI. He is also invited to present at conferences and audits in association with the following pharmaceutical companies: Vifor Pharma, UCB Pharma, Sanofi Aventis, Astra Zeneca, Glaxo Smith Kline and Pierre Fabre Med. Susanna Esposito declares that she has received research grants from Abbott, DMG, Glaxo Smith Kline, Merck, Sanofi and Vifor Pharma, and honoraria for advisory board and scientific presentations from Glaxo Smith Kline, Merck, Pfizer, Sanofi and Vifor Pharma. Susanna Esposito's new affiliation is Pietro Barilla Children's Hospital, Department of Medicine and Surgery, University of Parma, Parma, Italy.

Compliance with Ethics Guidelines. This article is based on previously conducted studies 
and does not contain any studies with human participants or animals performed by any of the authors.

Data Availability. Data sharing is not applicable to this article as no datasets were generated or analysed during the current study.

Open Access. This article is licensed under a Creative Commons Attribution-NonCommercial 4.0 International License, which permits any non-commercial use, sharing, adaptation, distribution and reproduction in any medium or format, as long as you give appropriate credit to the original author(s) and the source, provide a link to the Creative Commons licence, and indicate if changes were made. The images or other third party material in this article are included in the article's Creative Commons licence, unless indicated otherwise in a credit line to the material. If material is not included in the article's Creative Commons licence and your intended use is not permitted by statutory regulation or exceeds the permitted use, you will need to obtain permission directly from the copyright holder. To view a copy of this licence, visit http://creativecommons.org/licenses/by$\mathrm{nc} / 4.0 /$.

\section{REFERENCES}

1. Jain N, Lodha R, Kabra SK. Upper respiratory tract infections. Indian J Pediatr. 2001;68:1135-8.

2. Grief SN. Upper respiratory infections. Prim Care. 2013;40(3):757-70. https://doi.org/10.1016/j.pop. 2013.06.004 (Epub 2013 Jul 12).

3. Moellering RC Jr. The continuing challenge of lower respiratory tract infections. Clin Infect Dis. 2002;34(Suppl 1):S1-3.

4. Schaad UB, Esposito S, Razi CH. Diagnosis and management of recurrent respiratory tract infections in children: a practical guide. Arch Pediatr Infect Dis. 2016;4(1):e31039.

5. Baugh, et al. Clinical practice guideline: tonsillectomy in children. Otolaryngol Head Neck Surg. 2011;144(1 Suppl):S1-30. https://doi.org/10.1177/ 0194599810389949.
6. Schaad, et al. The management of recurrent respiratory tract infections in children. Eur Infect Dis. 2012;6(2):111-5.

7. West JV. Acute upper airway infections. Br Med Bull. 2002;61:215-30.

8. Esposito, et al. Circulation of different rhinovirus groups among children with lower respiratory tract infection in Kiremba, Burundi. Eur J Clin Microbiol Infect Dis. 2012;31:3251-6.

9. Jiang S, Sun L, Wang B, Yang X, Shang L, Zhang Y. Health-related quality of life among children with recurrent respiratory tract infections in $\mathrm{Xi}^{\prime}$ an China. PLoS One. 2013;8(2):e56945. https://doi. org/10.1371/journal.pone.0056945 (Epub 2013 Feb 25).

10. Shehab N, Patel PR, Srinivasan A, Budnitz DS. Emergency department visits for antibiotic-associated adverse events. Clin Infect Dis. 2008;47(6): 735-43. https://doi.org/10.1086/591126.

11. Neuzil KM, Hohlbein C, Zhu Y. Illness among schoolchildren during influenza season: effect on school absenteeism, parental absenteeism from work, and secondary illness in families. JAMA Pediatr. 2002;156(10):986-91.

12. Linder JA, Singer DE. Health-related quality of life of adults with upper respiratory tract infections. J Gen Intern Med. 2003;18(10):802-7.

13. Dethlefsen L, Huse S, Sogin ML, Relman DA. The pervasive effects of an antibiotic on the human gut microbiota, as revealed by deep 16S rRNA sequencing. PLoS Biol. 2008;6(11):e280. https://doi. org/10.1371/journal.pbio.0060280.

14. Kearney SC, Dziekiewicz M, Feleszko W. Immunoregulatory and immunostimulatory responses of bacterial lysates in respiratory infections and asthma. Ann Allergy Asthma Immunol. 2015;114(5):364-9. https://doi.org/10.1016/j.anai. 2015.02.008 (Epub 2015 Mar 6).

15. De Benedetto F, Sevieri G. Prevention of respiratory tract infections with bacterial lysate OM-85 bronchomunal in children and adults: a state of the art. Multidiscip Respir Med. 2013;8(1):33. https://doi. org/10.1186/2049-6958-8-33.

16. Esposito S, Soto-Martinez ME, Feleszko W, Jones $\mathrm{MH}$, Shen KL, Schaad UB. Nonspecific immunomodulators for recurrent respiratory tract infections, wheezing and asthma in children: a systematic review of mechanistic and clinical evidence. Curr Opin Allergy Clin Immunol. 2018;18(3):198-209. https://doi.org/10.1097/ACI. 0000000000000433. 
17. Gill N, Wlodarska M, Finlay BB. The future of mucosal immunology: studying an integrated system-wide organ. Nat Immunol. 2010;11:558-60.

18. Pfefferie PI, Prescott SL, Kopp M. Microbial influence on tolerance and opportunities for intervention with prebiotics/probiotics and bacterial lysates. J Allergy Clin Immunol. 2013;1321:1453-63.

19. Parola C, Salogni L, Vaira X, et al. Selective activation of human dendritic cells by OM-85 through a NF-kB and MAPK dependent pathway. PLoS One. 2013;8(12):e82867. https://doi.org/10.1371/ journal.pone.0082867 (eCollection 2013).

20. Dang AT, Pasquali C, Ludigs K, Guarda G. OM-85 is an immunomodulator of interferon- $\beta$ production and inflammasome activity. Sci Rep. 2017;7:43844. https://doi.org/10.1038/srep43844.

21. Pasquali C, Salami O, Taneja M, et al. Enhanced mucosal antibody production and protection against respiratory infections following an orally administered bacterial extract. Front Med (Lausanne). 2014;1:41. https://doi.org/10.3389/fmed. 2014.00041 (eCollection 2014).

22. Huber M, Mossmann H, Bessler WG. Th1-orientated immunological properties of the bacterial extract OM-85-BV. Eur J Med Res. 2005;10(5):209-17.

23. Rossi GA, Bessler W, Ballarini S, Pasquali C. Evidence that a primary anti-viral stimulation of the immune response by OM-85 reduces susceptibility to a secondary respiratory bacterial infection in mice. Ital J Pediatr. 2018;44(1):112. https://doi.org/ 10.1186/s13052-018-0569-7.

24. Tao Y, Yuan T, Li X, Yang S, Zhang F, Shi L. Bacterial extract OM-85 BV protects mice against experimental chronic rhinosinusitis. Int J Clin Exp Pathol. 2015;8(6):6800-6 (eCollection 2015).

25. Zagar S, Löfler-Badzek D. Broncho-Vaxom in children with rhinosinusitis: a double-blind clinical trial. ORL J Otorhinolaryngol Relat Spec. 1988;50(6):397-404.

26. Campbell H. Acute respiratory infection: a global challenge. Arch Dis Child. 1995;73(4):281-3.

27. Seemungal TA, Donaldson GC, Paul EA, Bestall JC, Jeffries DJ, Wedzicha JA. Effect of exacerbation on quality of life in patients with chronic obstructive pulmonary disease. Am J Respir Crit Care Med. 1998;157(5 Pt1):1418-22.

28. Arcavi L, Benowitz NL. Cigarette smoking and infection. Arch Intern Med. 2004;164(20):2206-16.

29. Paupe J. Immunotherapy with an oral bacterial extract (OM-85 BV) for upper respiratory infections. Respiration. 1991;58:150-4.
30. Del-Río-Navarro BE, Sienra-Monge JJL, Berber A, Torres-Alcántara S, Avila-Castañón L, Gómez-Barreto $\mathrm{D}$. Use of OM-85 BV in children suffering from recurrent respiratory tract infections and subnormal IgG subclass levels. Allergol Immunopathol (Madr). 2003;31:7-13.

31. Jara-Perez JV, Berber A. Primary prevention of acute respiratory tract infections in children using a bacterial immunostimulant: a double-masked, placebocontrolled clinical trial. Clin Ther. 2000;22:748-59.

32. Gutiérrez-Tarango MD, Berber A. Safety and efficacy of two courses of OM-85 BV in the prevention of respiratory tract infections in children during 12 months. Chest. 2001;119:1742-8.

33. Schaad UB. OM-85 BV, an immunostimulant in pediatric recurrent respiratory tract infections: a systematic review. World J Pediatr. 2010;6:5-12.

34. Del-Rio-Navarro BE, Espinosa-Rosales FJ, Flenady V. Sienra-Monge JJL Cochrane review: immunostimulants for preventing respiratory tract infection in children. Evid-Bas Child Health (Cochrane Review). 2012. https://doi.org/10.1002/ebch.1833.

35. Esposito S, Marchisio P, Prada E, et al. Impact of a mixed bacterial lysate $(\mathrm{OM}-85 \mathrm{BV})$ on the immunogenicity, safety and tolerability of inactivated influenza vaccine in children with recurrent respiratory tract infection. Vaccines. 2014;32: 2546-52. https://doi.org/10.1016/j.vaccine.2014. 03.055 (Epub 2014 Mar 26).

36. Razi CH, Harmanci $\mathrm{K}$, Abacı A, et al. The immunostimulant OM-85 BV prevents wheezing attacks in preschool children. J Allergy Clin Immunol. 2010;126(4):763-9. https://doi.org/10. 1016/j.jaci.2010.07.038.

37. Yin $\mathrm{J}, \mathrm{Xu} \mathrm{B}$, Zeng $\mathrm{X}$, Shen K. Broncho-Vaxom in pediatric recurrent respiratory tract infections: a systematic review and meta-analysis. Int Immunopharmacol. 2018;54:198-209.

38. Esposito S, Bianchini S, Polinori I, Principi N. Impact of OM-85 given during two consecutive years to children with a history of recurrent respiratory tract infections: a retrospective study. Int J Environ Res Public Health. 2019;16(6):E1065. https://doi.org/10.3390/ijerph16061065.

39. Esposito S, Bianchini S, Bosis S, et al. A randomized, placebo-controlled, double-blinded, single-centre, phase IV trial to assess the efficacy and safety of OM-85 in children suffering from recurrent respiratory tract infections. J Transl Med. 2019;17:284. https://doi.org/10.1186/s12967-019-2040-y.

40. Rosenfeld RM, Shin JJ, Schwartz SR, et al. Clinical practice guideline: otitis media with effusion 
(update). Otolaryngol Head Neck Surg. 2016;154(1

Suppl):S1-41. https://doi.org/10.1177/

0194599815623467.

41. Lusk R. Pediatric chronic rhinosinusitis. Curr Opin Otolaryngol Head Neck Surg. 2006;14(6):393-6.

42. Marengo, Juchli, Ardusso, Riolfi 1er Congreso mundial de ORL pediátrica Argentina.

43. Gómez Barreto D, De la Torre C, Alvarez A, Faure A, Berber A. [Safety and efficacy of OM-85-BV plus amoxicillin/clavulanate in the treatment of subacute sinusitis and the prevention of recurrent infections in children]. [Article in Spanish]. Allergol Immunopathol (Madr.) 1998;26(1):17-22.

44. Bitar MA, Saade R. The role of OM-85 BV (BronchoVaxom) in preventing recurrent acute tonsillitis in children. Int J Pediatr Otorhinolaryngol. 2013;77: 670-3.

45. Karaca NE, Gulez N, Aksu G, Azarsiz E, Kutukculer N. Does OM-85 BV prophylaxis trigger autoimmunity in IgA deficient children? Int Immunopharmacol. 2011;11:1747-51.

46. Del-Rio-Navarro BE, Espinosa Rosales F, Flenady V, Sienra-Monge JJ. Immunostimulants for preventing respiratory tract infection in children. Cochrane Database Syst Rev. 2006;4:CD004974.
47. Esposito S, Musio A. Immunostimulants and prevention of recurrent respiratory tract infections. J Biol Regul Homeost Agents. 2013;27(3):627-36.

48. Fokkens, et al. EPOS 2012: European position paper on rhinosinusitis and nasal polyps 2012. A summary for otorhinolaryngologists. Rhinology. 2012;50(1):1-12.

49. Dibildox-Martinez J, Mayorga Butron JL, Macias Fernandez LA, et al. Pan-American clinical guideline on rhinosinusitis. Otolaryngol Head Neck Surg. 2012;147(suppl 2):253. https://doi.org/10.1177/ $0194599812451426 a 408$.

50. Anselmo-Lima WT, Sakano E, Tamashiro E, et al. Rhinosinusitis: evidence and experience: October 18 and 19, 2013, São Paulo. Braz J Otorhinolaryngol. 2015;81(1 Suppl 1):S1-49. https://doi.org/10. 1016/j.bjorl.2015.01.003 (Erratum. In: Braz J Otorhinolaryngol. 2015;81(4):454).

51. Feleszko W, Marengo R, Vieira AS, Ratajczak K, Mayorga Butrón JL. Immunity-targeted approaches to the management of chronic and recurrent upper respiratory tract disorders in children. Clin Otolaryngol. 2019. https://doi.org/10.1111/coa.13335 (Epub ahead of print). 